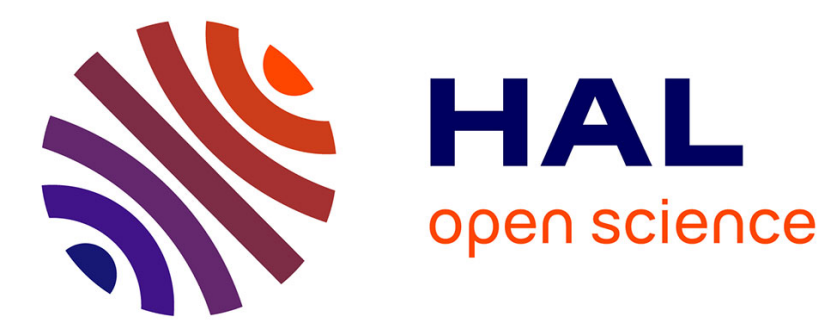

\title{
Robust statistical damage localization with stochastic load vectors
}

\author{
Luciano Marin, Michael Döhler, Dionisio Bernal, Laurent Mevel
}

\section{To cite this version:}

Luciano Marin, Michael Döhler, Dionisio Bernal, Laurent Mevel. Robust statistical damage localization with stochastic load vectors. Structural Control and Health Monitoring, 2015, 22 (3), pp.557-573. 10.1002/stc.1686 . hal-01063723

\section{HAL Id: hal-01063723 \\ https://inria.hal.science/hal-01063723}

Submitted on 5 Nov 2015

HAL is a multi-disciplinary open access archive for the deposit and dissemination of scientific research documents, whether they are published or not. The documents may come from teaching and research institutions in France or abroad, or from public or private research centers.
L'archive ouverte pluridisciplinaire HAL, est destinée au dépôt et à la diffusion de documents scientifiques de niveau recherche, publiés ou non, émanant des établissements d'enseignement et de recherche français ou étrangers, des laboratoires publics ou privés. 


\title{
Robust Statistical Damage Localization With Stochastic Load Vectors
}

\author{
Luciano Marin, Michael Döhler, Dionisio Bernal, Laurent Mevel
}

\begin{abstract}
The Stochastic Dynamic Damage Locating Vector approach is a vibrationbased damage localization method based on a finite element model of a structure and output-only measurements in both reference and damaged states. A stress field is computed for loads in the null space of a surrogate of the change in the transfer matrix at the sensor positions for some values in the Laplace domain. Then, the damage location is related to positions where the stress is close to zero. Robustness of the localization information can be achieved by aggregating results at different values in the Laplace domain. So far, this approach and in particular the aggregation is deterministic and does not take the uncertainty in the stress estimates into account. In this paper, the damage localization method is extended with a statistical framework. The uncertainty in the output-only measurements is propagated to the stress estimates at different values of the Laplace variable and these estimates are aggregated based on statistical principles. The performance of the new statistical approach is demonstrated both in a numerical application and a lab experiment, showing a significant improvement of the robustness of the method due to the statistical evaluation of the localization information.
\end{abstract}

\section{INTRODUCTION}

Over the last twenty years, linear system identification methods found a special interest in structural engineering for structural health monitoring. Using output-only measurements from structures in the field, Operational Modal Analysis (OMA) has been successfully used as a complementary technique to the traditional Experimental Modal Analysis (EMA) methods in the lab [1,2]. Sensors installed on the structures collect data, from which estimates of the modal parameters of interest (natural frequencies, damping ratios and mode shapes) can be obtained with methods originating from stochastic system realization theory for linear systems. Changes in these parameters indicate damage in a structure $[3,4]$, and damage localization is possible when linking these changes to a finite element model (FEM) of the structure. However, damage localization through FEM updating is often an ill-posed problem, since usually the dimension of the FEM is much larger than the number of identified modal parameters [5]. Alternative localization methods with a theoretical background, for example, 
account for the uniqueness issue by an appropriate re-parameterization (i.e. by clustering) and perform the localization with statistical tests [6-8]. Another class of methods interrogates changes in the flexibility of a structure [9-11], including the present one, and extract localization information based on the FEM without the need of a particular parameterization.

In particular, the Stochastic Dynamic Damage Location Vector (SDDLV) approach [12] is a damage localization technique using both finite element information and modal parameters from output-only vibration measurements. From estimates of the modes in both reference and damaged states, the null space of the difference between surrogates of the transfer matrices at the sensor positions for some Laplace variable $s$ in the complex plane is obtained. Then, a vector in this null space is applied as a load to the FEM of the structure in the reference state to compute the stress field. Damage is related to this stress field and it has been shown that potential damage locations are at elements where the stress is close to zero.

The modal parameter estimates from the output-only measurements that are used for damage localization are naturally subject to variance errors [13-15]. Taking into account these uncertainties proves to be important for structural damage diagnosis $[16,17]$. Based on these considerations, a statistical extension of the SDDLV method was developed in [18] for the actual decision if an element is damaged. This approach takes the intrinsic uncertainty of the stress computation associated to the measurements into account. Still, the Laplace domain variable $s$ needs to be chosen where the surrogate of the transfer function matrix difference is evaluated. Due to modal truncation, different choices of $s$ have a different impact on the localization results. Heuristic rules have been derived for the choice of these Laplace variables in [12], where a deterministic aggregation of results for different Laplace variables was suggested to increase the robustness of the approach. The main novelty of this paper is to move from the deterministic aggregation to a joint statistical evaluation for the different Laplace variables. In this way, it is expected to increase robustness of the damage localization approach since a part of the estimation errors and uncertainty related to the choice of the Laplace variables is removed. Moreover, this paper links the subspace-based modal parameter estimation [19-21] and its uncertainty quantification $[14,22]$ from output-only measurement data to the SDDLV-based stress computation and its statistical evaluation to give a complete and comprehensive damage localization algorithm.

This paper is organized as follows. First, the SDDLV approach is introduced as an output-only method for damage localization in Section 2. In Section 3 the subspace-based system identification step with the construction of parametric system matrices from identified modes and their uncertainty quantification is explained. Then, the robust statistical approach for the aggregation of damage localization results is derived in Section 4. In Section 5 the new method is successfully applied on numerical and real test cases. Finally, some conclusions of this work are presented in Section 6. 


\section{THE SDDLV APPROACH}

The SDDLV approach [12] is an output-only damage localization method based on interrogating changes $\delta G(s)$ in the transfer matrix $G(s)$ of a system, performed between two data sets: one from the undamaged (reference) state and another from the damaged state. Vectors in the null space of $\delta G(s)$ are obtained from system identification results using output-only measurements of both states. Then, they are applied as load vectors to a FEM for the computation of a stress field over the structure in order to indicate the damage location, since it has been shown that damaged elements induce zero stress (or close to zero in practice) $[9,12,23,24]$. To gain robustness in this localization approach, the stress field is computed for different values of the Laplace variable $s$ of the transfer matrix, and the results are aggregated. This section summarizes the deterministic computation of the stress field and the aggregation of the results, before we derive its statistical evaluation in Section 4 .

\section{$2.1 \quad$ Stress computation}

First, vectors in the null space of $\delta G(s)$ are computed using output-only measurements of the structure. The behavior of a mechanical structure is assumed to be described by a linear time-invariant (LTI) dynamical system

$$
M \ddot{\mathcal{X}}(t)+C \dot{\mathcal{X}}(t)+K \mathcal{X}(t)=v(t),
$$

where $t$ denotes continuous time, $M, C, K \in \mathbb{R}^{d \times d}$ are the mass, damping and stiffness matrices respectively and $\mathcal{X} \in \mathbb{R}^{d}$ collects the displacements of the $d$ degrees of freedom (DOF) of the structure. The external force $v(t)$ is unmeasured and modeled as white noise. Let the system (1) be observed at $r$ coordinates. As $v(t)$ is unmeasured, it can be replaced with a fictive force $e(t) \in \mathbb{R}^{r}$ acting only in the measured coordinates and that re-produces the measured output. With the substitution $x=\left[\begin{array}{ll}\mathcal{X}^{T} & \dot{\mathcal{X}}^{T}\end{array}\right]^{T}$ this leads to the corresponding continuous-time state-space model

$$
\left\{\begin{array}{l}
\dot{x}=A_{c} x+B_{c} e \\
y=C_{c} x+D_{c} e
\end{array}\right.
$$

with the state vector $x \in \mathbb{R}^{n}$, the output vector $y \in \mathbb{R}^{r}$, the state transition matrix $A_{c} \in \mathbb{R}^{n \times n}$ and the output mapping matrix $C_{c} \in \mathbb{R}^{r \times n}$, where $n$ is the system order and $r$ is the number of outputs. The input influence matrix and direct transmission matrix are $B_{c} \in \mathbb{R}^{n \times r}$ and $D_{c} \in \mathbb{R}^{r \times r}$, respectively. Only the system matrices $A_{c}$ and $C_{c}$ can be obtained from output-only system identification, which is described in detail in Section 3.

Depending on the kind of output measurements $y$ (displacements, velocities,

or accelerations; $b \stackrel{\text { def }}{=} 0,1,2$, respectively), the transfer matrix $G(s) \in \mathbb{C}^{r \times r}$ of system (2) can be expressed without the input influence matrix $B_{c}$ for $2 r \geq n$ as

$$
G(s)=R(s) D_{c}, \quad \text { with } \quad R(s)=C_{c} A_{c}^{-b}\left(s I-A_{c}\right)^{-1}\left[\begin{array}{c}
C_{c} A_{c}^{1-b} \\
C_{c} A_{c}^{-b}
\end{array}\right]^{\dagger}\left[\begin{array}{l}
I \\
0
\end{array}\right]
$$


as derived in detail in $[12,23]$, where $I$ denotes the identity matrix and ${ }^{\dagger}$ denotes the Moore-Penrose pseudoinverse. Note that the constraint $2 r \geq n$ is often inactive, since the number of identified modes is limited in practice. With the property $\left(A^{-b}\right)^{\dagger}=A^{b}$ and the relation $A_{c}^{-b}\left(s I-A_{c}\right)^{-1} A_{c}^{b}=\left(s I-A_{c}\right)^{-1}$, the expression for $R(s)$ in (3) is equivalent to

$$
R(s)=C_{c}\left(s I-A_{c}\right)^{-1}\left[\begin{array}{c}
C_{c} A_{c} \\
C_{c}
\end{array}\right]^{\dagger}\left[\begin{array}{l}
I \\
0
\end{array}\right]
$$

which is a helpful simplification and generalization. Equation (4) holds for displacement, velocity and acceleration measurements at the same time, unlike in [18] where only accelerations $(b=2)$ were considered. The corresponding simplified covariance expression for (4) will be derived in Section 4.3.

Let the variables in (3) be given in the damaged (variables with tilde) and reference states, respectively. The difference in the transfer matrices between both states is $\delta G(s)=\tilde{G}(s)-G(s)$. Then, assuming $\delta D_{c}=\tilde{D}_{c}-D_{c}=0$ (corresponding to no mass change) and $D_{c}$ being invertible, the matrices $\delta G(s)$ and $\delta R(s)^{T}=\tilde{R}(s)^{T}-R(s)^{T}$ have the same null space [12]. Thus, the desired load vector can be equivalently found in the null space of $\delta R(s)^{T}$, which can be obtained from the Singular Value Decomposition (SVD)

$$
\delta R(s)^{T}=U \Sigma V^{H}=\left[\begin{array}{ll}
U_{1} & U_{2}
\end{array}\right]\left[\begin{array}{cc}
\Sigma_{1} & 0 \\
0 & \Sigma_{2}
\end{array}\right]\left[\begin{array}{ll}
V_{1} & V_{2}
\end{array}\right]^{H},
$$

where $U, \Sigma, V \in \mathbb{C}^{r \times r}, \Sigma_{2} \approx 0$ and ${ }^{H}$ denotes the conjugate transpose. The desired load vector $v(s)$ in the null space of $\delta R(s)^{T}$ can be chosen as any linear combination of the vectors in $V_{2}$, in particular as the vector corresponding to the smallest singular value. Note that only output-only measurement data is necessary for the computation of an estimate of $v(s)$.

The computation of the stress from the load vector $v(s)$ implies knowledge of the finite element model (FEM) of the structure. Let $e$ be the number of all stresses that are computed at the elements of the FEM. First, displacements at all DOFs are computed from the load $v(s)$ at the sensor coordinates. Then, stress (or stress resultants) are computed at all the elements of the FEM from the displacements. The function corresponding to these operations is linear and denoted by the matrix $\mathcal{L}_{\text {model }}(s) \in \mathbb{C}^{e \times r}$, which is entirely obtained from the FEM of the structure $[12,18]$ and satisfies

$$
S(s)=\mathcal{L}_{\text {model }}(s) v(s)
$$

Theoretically, the components of the stress vector $S(s)$ corresponding to a damaged element are zero $[9,12]$ and hence entries in $S(s)$ close to zero indicate potentially (but not necessarily) damaged elements. In practice these stresses are not exactly zero but small due to modal truncation, model errors and uncertainties from the measurement data. Note that while the load vector $v(s)$ is only defined at the sensor coordinates, damage can be localized at any element of the structure because the stress vector generated from $v(s)$ covers the full domain. 


\subsection{Multiple stress vectors and aggregation}

Since damaged elements lead to stress values that are (close to) zero, but zero stress does not necessarily indicate damage [9] on one side, and due to truncation and model errors on the other side, it is recommended to compute the load vector $v(s)$ and subsequently the stress vector $S(s)$ for several values of the Laplace variable $s$. Robustness of the damage localization approach is then achieved by aggregating the results.

Let the Laplace variables $s_{i}, i=1, \ldots, \kappa$, be given. To minimize modal truncation errors, they should be chosen within a vicinity of the identified poles of the structure in the complex plane, but not too close to them [12]. After the identification of the system matrices $A_{c}$ and $C_{c}$ in the reference and $\tilde{A}_{c}$ and $\tilde{C}_{c}$ in the damaged states, the computations (4)-(6) are repeated for each value $s_{i}$ to obtain the respective stress vectors $S\left(s_{i}\right)$.

To decide if an element is damaged, the information of the corresponding entries in the stress vector $S\left(s_{i}\right)$ for all $i=1, \ldots, \kappa$ can be used now. In [12] the aggregation

$$
\bar{S}_{j}=\sum_{i=1}^{\kappa}\left|S_{j}\left(s_{i}\right)\right|
$$

for each entry $j$ was suggested. In Section 4.5 we propose a new aggregation scheme based on the uncertainties of the system identification results.

\section{STOCHASTIC SUBSPACE IDENTIFICATION (SSI) AND SYSTEM MODES SELECTION}

Estimates of the system matrices $A_{c}$ and $C_{c}$ from output-only measurements of the continuous-time system (2) are required for the damage localization strategy from the previous section. Stochastic subspace-based system identification methods are efficient tools for this task, identifying the discrete-time state-space model

$$
\left\{\begin{aligned}
x_{k+1} & =A_{d} x_{k}+v_{k} \\
y_{k} & =C_{d} x_{k}+w_{k}
\end{aligned}\right.
$$

where $A_{d}=\exp \left(A_{c} \tau\right), C_{d}=C_{c}, \tau$ is the time step and $v_{k}$ and $w_{k}$ are process and measurement noise, respectively. In the first step, we identify estimates $\widehat{A}_{d}$ and $\widehat{C}_{d}$ of the system matrices at different model orders from the measurements using covariance-driven subspace identification [19-21]. Due to noise, these model orders need to be relatively high and furthermore noise modes appear in the results. From these results at most $r$ identified mode pairs are selected in a so-called stabilization diagram in the second step, such that the condition $2 r \geq n$ is fulfilled. Finally, the corresponding eigenvalues of the continuous-time system and the mode shapes are used to build the desired estimates $\widehat{A}_{c}$ and $\widehat{C}_{c}$ in the last step, rejecting the noise modes. 


\subsection{Step 1: System identification}

For reference-based subspace identification a subset of the sensors can be selected in order to reduce the computation effort, the so-called reference sensors or projection channels [19] indicated by ${ }^{(\mathrm{ref})}$. Define the theoretical crosscorrelation between the state and the outputs $G \stackrel{\text { def }}{=} \mathbf{E}\left(x_{k+1} y_{k}^{(\text {ref }) T}\right)$, the output correlation $R_{i} \stackrel{\text { def }}{=} \mathbf{E}\left(y_{k} y_{k-i}^{(\text {ref }) T}\right)=C_{d} A_{d}^{i-1} G$ and the block Hankel matrix

$$
\mathcal{H} \stackrel{\text { def }}{=}\left[\begin{array}{cccc}
R_{1} & R_{2} & \ldots & R_{q} \\
R_{2} & R_{3} & \ldots & R_{q+1} \\
\vdots & \vdots & \ddots & \vdots \\
R_{p+1} & R_{p+2} & \ldots & R_{p+q}
\end{array}\right] \stackrel{\text { def }}{=} \operatorname{Hank}\left(R_{i}\right) .
$$

Matrix $\mathcal{H}$ possesses the well-known factorization property $\mathcal{H}=\mathcal{O C}$ into the matrices of observability and controllability

$$
\mathcal{O} \stackrel{\text { def }}{=}\left[\begin{array}{c}
C_{d} \\
C_{d} A_{d} \\
\vdots \\
C_{d} A_{d}^{p}
\end{array}\right] \text { and } \mathcal{C} \stackrel{\text { def }}{=}\left[\begin{array}{llll}
G & A_{d} G & \ldots & A_{d}^{q-1} G
\end{array}\right],
$$

respectively. From $\mathcal{O}$, the observation matrix $C_{d}$ is obtained in its first block row. Matrix $A_{d}$ is obtained from the shift invariance property of $\mathcal{O}$ as the least squares solution $A_{d}=\mathcal{O}^{\uparrow} \mathcal{O}^{\downarrow}$, where the last and the first block row has been removed in $\mathcal{O}^{\uparrow}$ and $\mathcal{O}^{\downarrow}$, respectively.

Using measurement data, the correlation estimates $\widehat{R}_{i}=1 / N \sum_{k=1}^{N} y_{k} y_{k-i}^{(\mathrm{ref}) T}$, $i=1, \ldots, p+q$, are computed to fill the Hankel matrix estimate $\widehat{\mathcal{H}}=\operatorname{Hank}\left(\widehat{R}_{i}\right)$ in (8). The observability matrix estimate $\widehat{\mathcal{O}}$ is obtained from a SVD of $\widehat{\mathcal{H}}$ and its truncation at the desired model order, and the system matrix estimates $\widehat{A}_{d}$ and $\widehat{C}_{d}$ are obtained as above. For simplicity, Sections 3.2 and 3.3 are written

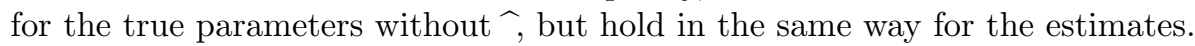

\subsection{Step 2: Mode selection}

From the system matrices $A_{d}$ and $C_{d}$, the eigenvalues $\lambda_{i}^{d}$ and mode shapes $\varphi_{i}$ for each mode $i$ are retrieved from

$$
\operatorname{det}\left(A_{d}-\lambda_{i}^{d} I\right)=0, \quad A_{d} \phi_{i}=\lambda_{i}^{d} \phi, \quad \varphi_{i}=C_{d} \phi_{i} .
$$

The eigenvalues $\lambda_{i}^{c}$ of the system matrix $A_{c}$ of the respective continuous-time system are then

$$
\lambda_{i}^{c}=\frac{1}{\tau} \ln \left(\lambda_{i}^{d}\right)
$$

and the natural frequencies $f_{i}$ and damping ratios $\xi_{i}$ are obtained from

$$
f_{i}=\frac{\left|\lambda_{i}^{c}\right|}{2 \pi \tau}, \quad \xi_{i}=\frac{-\operatorname{Re}\left(\lambda_{i}^{c}\right)}{\left|\lambda_{i}^{c}\right|}
$$


The set of identified modes does not contain only physical (true) modes of the structure in practice, but also spurious modes due to non-white noise and non-stationary excitation, a low signal-to-noise ratio, or a wrong selection of the model order. The standard technique for discriminating spurious from physical modes is the use of a stabilization diagram, where the modes are computed at multiple subsequent model orders and the identified frequencies are plotted versus the model order [19]. A fast multi-order computation for subspace identification was described in [21]. Finally, under the premise that the true system modes remain quite constant at different model orders, while spurious modes vary, the true structural modes can be chosen in the stabilization diagram based on user-defined stabilization criteria [25].

\subsection{Step 3: Final system matrices}

The system modes appear in conjugated complex pairs. Let $m$ be the number of mode pairs identified from the stabilization diagram, where $r \geq m$ to satisfy the constraint $2 r \geq n=2 m$. Let $\left(\lambda_{i}^{c}, \varphi_{i}\right)$ and $\left(\overline{\lambda_{i}^{c}}, \bar{\varphi}_{i}\right), i=1, \ldots, m$, be the identified modes. Then, the system matrices in the modal basis write as $A_{c}=$ $\operatorname{diag}\left(\lambda_{1}^{c}, \ldots, \lambda_{m}^{c}, \overline{\lambda_{1}^{c}}, \ldots, \overline{\lambda_{m}^{c}}\right)$ and $C_{c}=\left[\begin{array}{llll}\varphi_{1} & \ldots & \varphi_{m} & \bar{\varphi}_{1} \ldots \bar{\varphi}_{m}\end{array}\right]$. Since the system matrices $\left(A_{c}, C_{c}\right)$ are defined up to a change of basis $\left(T^{-1} A_{c} T, C_{c} T\right)$, we can equivalently define the real-valued system matrices

$$
A_{c}=\left[\begin{array}{cc}
\operatorname{Re}\left(\Lambda_{c}\right) & \operatorname{Im}\left(\Lambda_{c}\right) \\
-\operatorname{Im}\left(\Lambda_{c}\right) & \operatorname{Re}\left(\Lambda_{c}\right)
\end{array}\right], \quad C_{c}=[\operatorname{Re}(\Phi) \quad \operatorname{Im}(\Phi)]
$$

where $\Lambda_{c}=\operatorname{diag}\left(\lambda_{1}^{c}, \ldots, \lambda_{m}^{c}\right)$ and $\Phi=\left[\begin{array}{lll}\varphi_{1} & \ldots & \varphi_{m}\end{array}\right]$, obtained from the chosen modes.

\section{UNCERTAINTIES AND SDDLV ROBUST STATISTICAL TESTING}

For the SDDLV damage localization algorithm, estimates of the system matrices $A_{c}$ and $C_{c}$ are obtained in the reference and damaged states from a finite number of data samples. The identification of these matrices is subject to uncertainties due to the unknown excitation (being modeled as white noise), measurement noise and finite data length. Methods for the uncertainty quantification of the estimates from stochastic subspace identification in the context of structural vibration analysis are given in $[14,22,26]$.

Since the stress vector estimate $\widehat{S}\left(s_{i}\right)$ is computed from the estimates $\widehat{A}_{c}$ and $\widehat{C}_{c}$ in both reference and damaged states, the uncertainties from the system identification are propagated to uncertainties in the stress vector. These uncertainties may be crucial in deciding whether an element is potentially damaged, i.e. whether the stress of an element is in fact zero or not, while the computed stress value is just close to zero. In [18] the uncertainty propagation from sys-

tem identification results in general to the stress $\widehat{S}\left(s_{i}\right)$ for a single value of the 
Laplace variable $s_{i}$ was analyzed. In this section, the uncertainty propagation is made explicit for the chosen subspace identification algorithm from Section 3. Furthermore, expressions for the uncertainty propagation to the stress vector $\widehat{S}\left(s_{i}\right)$ computed at multiple values $s_{i}, i=1, \ldots, \kappa$, are derived. With these means, the aggregation of the results and the test for damaged elements can be performed based on statistical criteria. Finally, the respective test is derived.

\subsection{Principles of covariance computation and uncertainty propagation}

The uncertainties in the measurement data are propagated to the damage localization results. The former can be quantified by a covariance estimate $\widehat{\Sigma}_{\mathcal{H}}=$ $\operatorname{cov}(\operatorname{vec}(\widehat{\mathcal{H}}))$ of the Hankel matrix, where $\operatorname{vec}(\cdot)$ denotes the column stacking vectorization operator. The estimate $\widehat{\Sigma}_{\mathcal{H}}$ can be directly estimated from the data as described in [14,22] by separating the available data of length $N$ into $n_{b}$ blocks of length $N_{b}$, such that $N=n_{b} N_{b}$. Then, the Hankel matrix $\widehat{\mathcal{H}}^{(j)}=\operatorname{Hank}\left(\widehat{R}_{i}^{(j)}\right)$ in (8) is computed on the correlations $\widehat{R}_{i}^{(j)}=1 / N_{b} \sum_{k=1+(j-1) N_{b}}^{j N_{b}} y_{k} y_{k-i}^{(\mathrm{ref}) T}$ on each block. The Hankel matrix on the entire dataset hence yields $\widehat{\mathcal{H}}=\frac{1}{n_{b}} \sum_{j=1}^{n_{b}} \widehat{\mathcal{H}}^{(j)}$, and the covariance estimate

$$
\widehat{\Sigma}_{\mathcal{H}}=\frac{1}{n_{b}-1} \sum_{j=1}^{n_{b}} \operatorname{vec}\left(\widehat{\mathcal{H}}^{(j)}-\widehat{\mathcal{H}}\right) \operatorname{vec}\left(\widehat{\mathcal{H}}^{(j)}-\widehat{\mathcal{H}}\right)^{T} .
$$

is obtained.

All computed quantities in Sections 2 and 3 are ultimately functions of the Hankel matrix. Let $f$ be such a vector valued function of $\widehat{\mathcal{H}}$. Its covariance can be calculated using the Taylor approximation as

$$
f(\widehat{\mathcal{H}}) \approx f(\mathcal{H})+\mathcal{J}_{f, \mathcal{H}} \operatorname{vec}(\widehat{\mathcal{H}}-\mathcal{H}) \Rightarrow \operatorname{cov}(f(\widehat{\mathcal{H}})) \approx \mathcal{J}_{f, \mathcal{H}} \widehat{\Sigma}_{\mathcal{H}} \mathcal{J}_{f, \mathcal{H}}^{T}
$$

with the sensitivity matrix $\mathcal{J}_{f, \mathcal{H}} \stackrel{\text { def }}{=} \partial f(\mathcal{H}) / \partial \operatorname{vec}(\mathcal{H})$, which can be obtained analytically $[13,14,18,22]$. A consistent estimate is obtained by replacing the theoretical variables (such as $\mathcal{H}$ ) with consistent estimates obtained from the data (such as $\widehat{\mathcal{H}}$ ) in the computation of the sensitivity matrix. With the operator $\Delta$ we define (theoretical) first-order perturbations, yielding $\Delta f=\mathcal{J}_{f, \mathcal{H}} \operatorname{vec}(\Delta \mathcal{H})$. Using this relationship, the desired sensitivity matrix can be obtained and used for the covariance computation in (13). Like this, the covariance of the Hankel matrix can be propagated to any parameters that are dependent on the data, in particular to the modal parameters and the stress estimate $\widehat{S}\left(s_{i}\right)$.

In the following, the sensitivity and covariance computation is done for the theoretical values (like $\mathcal{H}, A_{c}, C_{c}, S\left(s_{i}\right)$ ). Estimates of the sensitivities and covariances are then obtained by plugging in the estimates obtained from the data (like $\widehat{\mathcal{H}}, \widehat{A}_{c}, \widehat{C}_{c}, \widehat{S}\left(s_{i}\right)$ ).

The following notation and properties will be used. $\otimes$ denotes the Kronecker product, having the property $\operatorname{vec}(A X B)=\left(B^{T} \otimes A\right) \operatorname{vec}(X) . \quad I_{a}$ denotes the 
identity matrix of size $a \times a$, and $0_{a, b}$ denotes the zero matrix of size $a \times b$. $e_{j}^{a} \in \mathbb{R}^{a}$ denotes the $j$-th unit vector (being column $j$ of $I_{a}$ ). The permutation matrix $\mathcal{P}_{a, b} \stackrel{\text { def }}{=}\left[\begin{array}{llll}I_{a} \otimes e_{1}^{b} & I_{a} \otimes e_{2}^{b} & \ldots & I_{a} \otimes e_{b}^{b}\end{array}\right] \in \mathbb{R}^{a b \times a b}$ is defined with the property

$$
\operatorname{vec}\left(X^{T}\right)=\mathcal{P}_{a, b} \operatorname{vec}(X)
$$

for any matrix $X \in \mathbb{R}^{a \times b}[22]$. Finally, for dealing with the uncertainties of complex-valued matrices we introduce an equivalent real-valued notation by defining

$$
M_{\mathrm{Re}} \stackrel{\text { def }}{=}\left[\begin{array}{cc}
\operatorname{Re}(M) & -\operatorname{Im}(M) \\
\operatorname{Im}(M) & \operatorname{Re}(M)
\end{array}\right], \quad M_{\mathrm{re}} \stackrel{\text { def }}{=}\left[\begin{array}{l}
\operatorname{Re}(M) \\
\operatorname{Im}(M)
\end{array}\right]
$$

for any matrix $M$ as in $[13,18]$. Then, for example, a complex-valued equation $A x=b$ is equivalent to $A_{\mathrm{Re}} x_{\mathrm{re}}=b_{\mathrm{re}}$, and the sensitivities of the real-valued matrices can be derived.

\subsection{Covariance of system matrices $A_{c}$ and $C_{c}$ from sub- space identification}

For the covariance computation of $A_{c}$ and $C_{c}$, a perturbation $\Delta \mathcal{H}$ of the Hankel matrix in (8) is propagated to a perturbation $\Delta \lambda_{i}^{c}$ and $\Delta \varphi_{i}$ in the selected modes $i=1, \ldots, m$ (see Section 3.2), yielding

$$
\Delta \lambda_{i}^{c}=\mathcal{J}_{\lambda_{i}^{c}, \mathcal{H}} \operatorname{vec}(\Delta \mathcal{H}), \quad \Delta \varphi_{i}=\mathcal{J}_{\varphi_{i}, \mathcal{H}} \operatorname{vec}(\Delta \mathcal{H}),
$$

where the sensitivities $\mathcal{J}_{\lambda_{i}^{c}, \mathcal{H}} \in \mathbb{C}^{1 \times h}$ and $\mathcal{J}_{\varphi_{i}, \mathcal{H}} \in \mathbb{C}^{r \times h}$ with $h=(p+1) q r r^{(\mathrm{ref})}$ have been derived in detail in $[14,22]$. Plugging these expressions into (12) and vectorizing the system matrices leads to

$$
\operatorname{vec}\left(\Delta A_{c}\right)=\mathcal{J}_{A_{c}, \mathcal{H}} \operatorname{vec}(\Delta \mathcal{H}), \quad \operatorname{vec}\left(\Delta C_{c}\right)=\mathcal{J}_{C_{c}, \mathcal{H}} \operatorname{vec}(\Delta \mathcal{H}),
$$

where

$$
\mathcal{J}_{A_{c}, \mathcal{H}}=P_{1}\left[\begin{array}{c}
\operatorname{Re}\left(\mathcal{J}_{\lambda_{1}^{c}, \mathcal{H}}\right) \\
\vdots \\
\operatorname{Re}\left(\mathcal{J}_{\lambda_{m}^{c}, \mathcal{H}}\right) \\
\operatorname{Im}\left(\mathcal{J}_{\lambda_{1}^{c}, \mathcal{H}}\right) \\
\vdots \\
\operatorname{Im}\left(\mathcal{J}_{\lambda_{m}^{c}, \mathcal{H}}\right)
\end{array}\right], \mathcal{J}_{C_{c}, \mathcal{H}}=\left[\begin{array}{c}
\operatorname{Re}\left(\mathcal{J}_{\varphi_{1}, \mathcal{H}}\right) \\
\vdots \\
\operatorname{Re}\left(\mathcal{J}_{\varphi_{m}, \mathcal{H}}\right) \\
\operatorname{Im}\left(\mathcal{J}_{\varphi_{1}, \mathcal{H}}\right) \\
\vdots \\
\operatorname{Im}\left(\mathcal{J}_{\varphi_{m}, \mathcal{H}}\right)
\end{array}\right], P_{1}=\left[\begin{array}{c}
E_{1} \\
\vdots \\
E_{m} \\
F_{1} \\
\vdots \\
F_{m}
\end{array}\right]
$$

and the selection matrix $P_{1}$ is composed of the matrices

$$
E_{j}=\left[\begin{array}{cc}
e_{j}^{m} e_{j}^{m T} & 0_{m, m} \\
0_{m, m} & -e_{j}^{m} e_{j}^{m T}
\end{array}\right], F_{j}=\left[\begin{array}{cc}
0_{m, m} & e_{j}^{m} e_{j}^{m T} \\
e_{j}^{m} e_{j}^{m T} & 0_{m, m}
\end{array}\right] .
$$

Then, the covariance of the system matrices can be obtained from (16) as

$$
\Sigma_{A_{c}, C_{c}} \stackrel{\text { def }}{=} \operatorname{cov}\left(\left[\begin{array}{c}
\operatorname{vec}\left(A_{c}\right) \\
\operatorname{vec}\left(C_{c}\right)
\end{array}\right]\right)=\left[\begin{array}{c}
\mathcal{J}_{A_{c}, \mathcal{H}} \\
\mathcal{J}_{C_{c}, \mathcal{H}}
\end{array}\right] \Sigma_{\mathcal{H}}\left[\begin{array}{c}
\mathcal{J}_{A_{c}, \mathcal{H}} \\
\mathcal{J}_{C_{c}, \mathcal{H}}
\end{array}\right]^{T} .
$$




\subsection{Sensitivity of $R(s)$}

In the next step, the sensitivity $\mathcal{J}_{R(s), A_{c}, C_{c}}$ of the matrix $R(s)$ with respect to the system matrices $A_{c}$ and $C_{c}$ is derived, which is needed for the stress computation in (5)-(7). In the following we generalize the sensitivity computation for acceleration data in [18] to the simplified and more general formula of $R(s)$ in (4). Define

$$
Z(s)=C_{c}\left(s I-A_{c}\right)^{-1}, \quad H=\left[\begin{array}{c}
C_{c} A_{c} \\
C_{c}
\end{array}\right], \quad L=\left[\begin{array}{l}
I \\
0
\end{array}\right]
$$

such that $R(s)=Z(s) H^{\dagger} L$. Applying the product rule, a first-order perturbation of $R(s)$ yields

$$
\Delta R(s)=[\Delta Z(s)] H^{\dagger} L+Z(s)\left[\Delta\left(H^{\dagger}\right)\right] L .
$$

Both terms $\Delta Z(s)$ and $\Delta\left(H^{\dagger}\right)$ are now developed as functions of $\Delta A_{c}$ and $\Delta C_{c}$, which are already given in (16).

Using the relation $\Delta\left(X^{-1}\right)=-X^{-1}[\Delta X] X^{-1}$, the first term yields

$$
\begin{aligned}
\Delta Z(s) & =\left[\Delta C_{c}\right]\left(s I-A_{c}\right)^{-1}-C_{c}\left(s I-A_{c}\right)^{-1}\left[\Delta\left(s I-A_{c}\right)\right]\left(s I-A_{c}\right)^{-1} \\
& =\left[\Delta C_{c}\right]\left(s I-A_{c}\right)^{-1}+Z(s)\left[\Delta A_{c}\right]\left(s I-A_{c}\right)^{-1}
\end{aligned}
$$

For the second term, the perturbation of the pseudoinverse $H^{\dagger}$ yields

$$
\operatorname{vec}\left(\Delta\left(H^{\dagger}\right)\right)=\mathcal{J}_{H^{\dagger}} \operatorname{vec}(\Delta H),
$$

where the sensitivity $\mathcal{J}_{H^{\dagger}}$ is given in [18, Lemma 8]. Define the selection matrices $S_{1} \stackrel{\text { def }}{=} I_{n} \otimes\left[\begin{array}{ll}I_{r} & 0_{r, r}\end{array}\right], S_{2} \stackrel{\text { def }}{=} I_{n} \otimes\left[\begin{array}{ll}0_{r, r} & I_{r}\end{array}\right]$ such that

$$
\operatorname{vec}(\Delta H)=\left[\begin{array}{ll}
S_{1}^{T} & S_{2}^{T}
\end{array}\right]\left[\begin{array}{c}
\operatorname{vec}\left(\Delta\left(C_{c} A_{c}\right)\right) \\
\operatorname{vec}\left(\Delta C_{c}\right)
\end{array}\right]=S_{1}^{T} \operatorname{vec}\left(\Delta\left(C_{c} A_{c}\right)\right)+S_{2}^{T} \operatorname{vec}\left(\Delta C_{c}\right),
$$

and together with (20)

$$
\operatorname{vec}\left(\Delta\left(H^{\dagger}\right)\right)=\mathcal{J}_{H^{\dagger}} S_{1}^{T} \operatorname{vec}\left(\Delta\left(C_{c} A_{c}\right)\right)+\mathcal{J}_{H^{\dagger}} S_{2}^{T} \operatorname{vec}\left(\Delta C_{c}\right),
$$

where $\Delta\left(C_{c} A_{c}\right)=\left[\Delta C_{c}\right] A_{c}+C_{c}\left[\Delta A_{c}\right]$. Combining this result with (18) and (19) yields subsequently

$$
\operatorname{vec}(\Delta R(s))=\left[\begin{array}{ll}
\mathcal{J}_{R(s), A_{c}} & \mathcal{J}_{R(s), C_{c}}
\end{array}\right]\left[\begin{array}{c}
\operatorname{vec}\left(\Delta A_{c}\right) \\
\operatorname{vec}\left(\Delta C_{c}\right)
\end{array}\right]
$$

where

$$
\begin{aligned}
& \mathcal{J}_{R(s), A_{c}} \stackrel{\text { def }}{=}\left(M(s)^{T} \otimes Z(s)\right)+\left(L^{T} \otimes Z(s)\right) \mathcal{J}_{H^{\dagger}} S_{1}^{T}\left(I_{n} \otimes C_{c}\right), \\
& \mathcal{J}_{R(s), C_{c}} \stackrel{\text { def }}{=}\left(M(s)^{T} \otimes I_{r}\right)+\left(L^{T} \otimes Z(s)\right) \mathcal{J}_{H^{\dagger}}\left(S_{1}^{T}\left(A_{c}^{T} \otimes I_{r}\right)+S_{2}^{T}\right),
\end{aligned}
$$


with $M(s) \stackrel{\text { def }}{=}\left(s I-A_{c}\right)^{-1} H^{\dagger} L$. Stacking the real and imaginary parts of $\operatorname{vec}\left(\Delta R(s)^{T}\right)$, it follows from (14) and (15)

$$
\left(\operatorname{vec}\left(\Delta R(s)^{T}\right)\right)_{\mathrm{re}}=\mathcal{J}_{R(s)}\left[\begin{array}{c}
\operatorname{vec}\left(\Delta A_{c}\right) \\
\operatorname{vec}\left(\Delta C_{c}\right)
\end{array}\right]
$$

where

$$
\mathcal{J}_{R(s)}=\left[\begin{array}{cc}
\mathcal{P}_{r, r} & 0_{r^{2}, r^{2}} \\
0_{r^{2}, r^{2}} & \mathcal{P}_{r, r}
\end{array}\right]\left[\begin{array}{ll}
\operatorname{Re}\left(\mathcal{J}_{R(s), A_{c}}\right) & \operatorname{Re}\left(\mathcal{J}_{R(s), C_{c}}\right) \\
\operatorname{Im}\left(\mathcal{J}_{R(s), A_{c}}\right) & \operatorname{Im}\left(\mathcal{J}_{R(s), C_{c}}\right)
\end{array}\right]
$$

\subsection{Covariance of stress vector $S(s)$}

With the computations in the previous section, the covariance associated to the matrices $R(s)$ and $\tilde{R}(s)$ can be obtained in the reference and damaged states of the structure for a chosen value $s$. In the next step, these covariances are propagated to the load vector $v(s)$ in the null space of $\delta R(s)^{T}=\tilde{R}(s)^{T}-R(s)^{T}$ and finally to the stress vector $S(s)$, as computed in (5)-(6).

The covariance propagation to the null space vector $v(s)$ is based on the relation $\Delta v(s)_{\text {re }}=\mathcal{J}_{v(s)}\left(\operatorname{vec}\left(\Delta \delta R(s)^{T}\right)\right)_{\text {re }}$, where $\mathcal{J}_{v(s)}$ is derived in [18, Prop. 4]. Then, from (6) it follows

$$
\Delta S(s)_{\mathrm{re}}=\mathcal{J}_{S(s)}\left(\operatorname{vec}\left(\Delta \delta R(s)^{T}\right)\right)_{\mathrm{re}},
$$

where $\mathcal{J}_{S(s)}=\mathcal{L}_{\text {model }}(s)_{\operatorname{Re}} \mathcal{J}_{v(s)}$, and together with (21) it follows

$$
\Delta S(s)_{\mathrm{re}}=\mathcal{J}_{S(s)} \mathcal{J}_{\tilde{R}(s)}\left[\begin{array}{c}
\operatorname{vec}\left(\Delta \tilde{A}_{c}\right) \\
\operatorname{vec}\left(\Delta \tilde{C}_{c}\right)
\end{array}\right]-\mathcal{J}_{S(s)} \mathcal{J}_{R(s)}\left[\begin{array}{c}
\operatorname{vec}\left(\Delta A_{c}\right) \\
\operatorname{vec}\left(\Delta C_{c}\right)
\end{array}\right] .
$$

Since the system matrices from the damaged and reference states are obtained on different statistically independent measurements, the covariance expression

$\Sigma_{S(s)} \stackrel{\text { def }}{=} \operatorname{cov}\left(S(s)_{\text {re }}\right)=\mathcal{J}_{S(s)} \mathcal{J}_{\tilde{R}(s)} \Sigma_{\tilde{A}_{c}, \tilde{C}_{c}} \mathcal{J}_{\tilde{R}(s)}^{T} \mathcal{J}_{S(s)}^{T}+\mathcal{J}_{S(s)} \mathcal{J}_{R(s)} \Sigma_{A_{c}, C_{c}} \mathcal{J}_{R(s)}^{T} \mathcal{J}_{S(s)}^{T}$

follows, analogously to [18, Thm. 5].

\subsection{Statistical aggregation and evaluation of stress results at multiple $s$-values}

Since the computation of the stress $S\left(s_{i}\right)$ at multiple Laplace variables $s_{i}, i=$ $1, \ldots, \kappa$, increases the information content on the damage location (see Section 2.2 ), a joint evaluation of these stresses increases the robustness of the statistical approach in [18], where only one Laplace variable $s$ was considered. The joint statistical evaluation requires the covariance computation of the stacked (realvalued) stress results

$$
\bar{S} \stackrel{\text { def }}{=}\left[\begin{array}{c}
S\left(s_{1}\right)_{\mathrm{re}} \\
\vdots \\
S\left(s_{\kappa}\right)_{\mathrm{re}}
\end{array}\right] .
$$


Stacking (22) for each $s_{i}$ accordingly leads to

$$
\Delta \bar{S}=\left[\begin{array}{c}
\mathcal{J}_{S\left(s_{1}\right)} \mathcal{J}_{\tilde{R}\left(s_{1}\right)} \\
\vdots \\
\mathcal{J}_{S\left(s_{\kappa}\right)} \mathcal{J}_{\tilde{R}\left(s_{\kappa}\right)}
\end{array}\right]\left[\begin{array}{l}
\operatorname{vec}\left(\Delta \tilde{A}_{c}\right) \\
\operatorname{vec}\left(\Delta \tilde{C}_{c}\right)
\end{array}\right]-\left[\begin{array}{c}
\mathcal{J}_{S\left(s_{1}\right)} \mathcal{J}_{R\left(s_{1}\right)} \\
\vdots \\
\mathcal{J}_{S\left(s_{\kappa}\right)} \mathcal{J}_{R\left(s_{\kappa}\right)}
\end{array}\right]\left[\begin{array}{l}
\operatorname{vec}\left(\Delta A_{c}\right) \\
\operatorname{vec}\left(\Delta C_{c}\right)
\end{array}\right],
$$

and, analogously to (23), to the covariance $\Sigma_{\bar{S}} \stackrel{\text { def }}{=} \operatorname{cov}(\bar{S})$ with

$\Sigma_{\bar{S}}=\left[\begin{array}{c}\mathcal{J}_{S\left(s_{1}\right)} \mathcal{J}_{\tilde{R}\left(s_{1}\right)} \\ \vdots \\ \mathcal{J}_{S\left(s_{\kappa}\right)} \mathcal{J}_{\tilde{R}\left(s_{\kappa}\right)}\end{array}\right] \Sigma_{\tilde{A}_{c}, \tilde{C}_{c}}\left[\begin{array}{c}\mathcal{J}_{S\left(s_{1}\right)} \mathcal{J}_{\tilde{R}\left(s_{1}\right)} \\ \vdots \\ \mathcal{J}_{S\left(s_{\kappa}\right)} \mathcal{J}_{\tilde{R}\left(s_{\kappa}\right)}\end{array}\right]^{T}+\left[\begin{array}{c}\mathcal{J}_{S\left(s_{1}\right)} \mathcal{J}_{R\left(s_{1}\right)} \\ \vdots \\ \mathcal{J}_{S\left(s_{\kappa}\right)} \mathcal{J}_{R\left(s_{\kappa}\right)}\end{array}\right] \Sigma_{A_{c}, C_{c}}\left[\begin{array}{c}\mathcal{J}_{S\left(s_{1}\right)} \mathcal{J}_{R\left(s_{1}\right)} \\ \vdots \\ \mathcal{J}_{S\left(s_{\kappa}\right)} \mathcal{J}_{R\left(s_{\kappa}\right)}\end{array}\right]^{T}$.

While in previous works [12] the stress values $S\left(s_{i}\right)$ at multiple Laplace variables were aggregated empirically as in (7), the covariance expression (25) allows a new statistical aggregation scheme for damage localization based on a statistical test for each element $j$ of the structure. In this scheme, all stress values at the different Laplace variables in $\bar{S}$ corresponding to an element $j$ are tested for being 0 (and thus possibly damaged) in a hypothesis test, where the computed stress values are pondered individually by their covariance. A scalar test variable $\chi_{j}^{2}$ is computed for each element $j$ in such a test as follows.

First, the selection matrix for retrieving all entries in vector $\bar{S}$ corresponding to an element $j$ is described. Let $T_{j} \in \mathbb{R}^{n_{j} \times e}$ be the matrix that selects all entries computed for an element $j$ in the stress vector $S(s)$. The number $n_{j}$ of these stress resultants for an element $j$ depends on the kind of the element and the way of the computation of the stress resultant(s), which is up to the user. Accounting for the real and imaginary parts of the stress vector, and the stacking for all Laplace variables in $(24)$, the vector $\bar{S}_{j}$ containing all stress values corresponding to an element $j$ is

$$
\bar{S}_{j}=P_{j} \bar{S} \in \mathbb{R}^{2 \kappa n_{j}} \quad \text { where } \quad P_{j}=I_{\kappa} \otimes\left[\begin{array}{cc}
T_{j} & 0_{n_{j}, e} \\
0_{n_{j}, e} & T_{j}
\end{array}\right] .
$$

Second, the covariance of vector $\bar{S}_{j}$ is selected from $\Sigma_{\bar{S}}$ accordingly as

$$
\Sigma_{j}=P_{j} \Sigma_{\bar{S}} P_{j}^{T} .
$$

Since an estimate of vector $\bar{S}_{j}$ is asymptotically Gaussian distributed [18], it can finally be tested for being 0 and thus potentially damaged with the $\chi^{2}$-test variable

$$
\chi_{j}^{2}=\bar{S}_{j}^{T} \Sigma_{j}^{-1} \bar{S}_{j}
$$

which has $2 \kappa n_{j}$ degrees of freedom and non-centrality parameter 0 in the damaged case. Thus, the scalar variable $\chi_{j}^{2}$ contains a statistically meaningful aggregation of all stress results computed for an element $j$, and it can be decided if an element $j$ is potentially damaged if $\chi_{j}^{2}$ is below a threshold. Such a threshold 
can be theoretically obtained from the properties of the $\chi^{2}$-distribution for a desired type I or type II error, but for practical purposes it is often sufficient to announce potential damage locations in the elements $j$ with the lowest values of $\chi_{j}^{2}$.

\section{APPLICATIONS}

The damage localization algorithm with the statistical aggregation of the SDDLV results from Section 4.5 has been applied on two applications of increasing difficulty, first on a numerical simulation of a plate and second on measurements of a real beam in a lab experiment. For each application, the statistical aggregation of the computed stresses in Equation (26) from Section 4.5 is compared to the previously suggested deterministic aggregation in Equation (7) from Section 2.2. Stresses close to zero correspond to potentially damaged elements.

The different Laplace variables ( $s$-values) considered in these applications are empirically chosen within the range of the identified poles as suggested in [12]. For both applications, the presented results include the information coming from SDDLV at all different $s$-values mixed with the respective aggregations. Since damage localization with these methods corresponds to finding the elements with the lowest aggregated stress values, the absolute values of (7) and (26) are limited to some level to increase the scale contrast on the potentially damaged elements. All stress results are then normalized to a scale between 0 (possibly damaged, red) and 1 (not damaged, blue) for a visual presentation. The black box or oval in the figures denotes the damaged region.

\subsection{Simulated plate}

\subsubsection{Plate model and data simulation}

The considered plate model has the dimensions $150 \mathrm{~cm} \times 100 \mathrm{~cm} \times 1 \mathrm{~cm}$. Its edges are fixed and it consists of 100 plate elements as depicted in Figure 1. Damage in the plate was simulated by decreasing the stiffness in element 66 in two steps by $30 \%$ and $50 \%$ of its original value. For the undamaged and all damaged states, acceleration data of length $N=100,000$ with a sampling frequency of $600 \mathrm{~Hz}$ was generated at twelve sensor positions as shown in Figure 1. The output-only data was generated from collocated white noise excitation at the sensor positions, and $1 \%$ white measurement noise were added on the outputs.

\subsubsection{Modal analysis and uncertainties}

From the subspace-based system identification as described in Section 3, nine well-estimated modes were chosen for each structural state in the respective stabilization diagrams. Then, matrices $\widehat{A}_{c}$ and $\widehat{C}_{c}$ in both the undamaged and damaged states are filled and their uncertainty is obtained from the same dataset 
Table 1: Identified frequencies (in $\mathrm{Hz}$ ) of simulated plate with coefficients of variation (in \%).

\begin{tabular}{r|rrrrrrrrr}
\hline state & 1 & 2 & 3 & 4 & 5 & 6 & 7 & 8 & 9 \\
\hline reference & 34.43 & 65.93 & 105.7 & 118.5 & 135.5 & 185.3 & 191.6 & 224.4 & 284.5 \\
& 0.08 & 0.07 & 0.06 & 0.06 & 0.06 & 0.06 & 0.06 & 0.06 & 0.08 \\
\hline $30 \%$ damage & 34.31 & 65.85 & 105.3 & 118.0 & 135.2 & 185.0 & 191.3 & 224.2 & 283.8 \\
& 0.09 & 0.07 & 0.06 & 0.06 & 0.06 & 0.05 & 0.06 & 0.06 & 0.10 \\
\hline $50 \%$ damage & 34.18 & 65.71 & 105.0 & 117.8 & 135.2 & 184.4 & 191.1 & 224.0 & 283.6 \\
& 0.09 & 0.08 & 0.06 & 0.06 & 0.06 & 0.06 & 0.06 & 0.06 & 0.09 \\
\hline
\end{tabular}

as described in Sections 4.1-4.2. The summary of all underlying identified frequencies with their uncertainties is given in Table 1 . Note that the frequency decrease due to damage is less than $0.7 \%$ in the $30 \%$ damage case and less than $0.8 \%$ in the $50 \%$ damage case.

\subsubsection{Stress computation and evaluation}

In a preliminary step, the matrix $\mathcal{L}_{\text {model }}(s)$ in $(6)$ is obtained from the FEM of the plate model, such that the moments $m_{x x}$ and $m_{y y}$ are computed as the stress resultants for each of the 100 plate elements from the load vector at the sensor coordinates. The Laplace variables were chosen as $s_{1}=0, s_{2}=1+40 \mathrm{i}$, $s_{3}=1+140 \mathrm{i}, s_{4}=1+250 \mathrm{i}, s_{5}=1+400 \mathrm{i}$ and $s_{6}=1+800 \mathrm{i}$. Then, the real and imaginary parts of the stress vector $S\left(s_{i}\right)$ containing these moments and their joint covariance in (25) is computed for these Laplace variables.

Thus, 24 stress related values are computed for each plate element $j$ (real and imaginary parts of 2 moments at 6 Laplace variables). These values are aggregated statistically in the variable $\chi_{j}^{2}$ in (26) for each element $j$ with the new

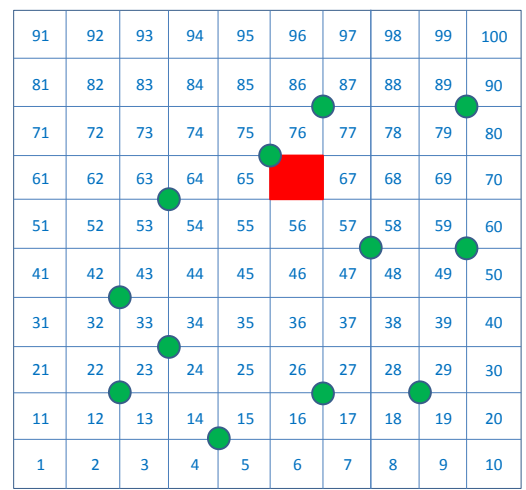

Figure 1: Geometry of the plate with twelve sensor positions and the damaged element. 
method described in this paper. Also, the previous deterministic aggregation of these values in (7) is performed for a comparison.

To illustrate the difficulties in the stress computation for damage localization that are related to modal truncation (using only nine modes) and estimation errors (uncertainties on modes), the following computations are made in the numerical examples:

(a) Exact stress: based on the theoretic modes obtained from the models. Contains only modal truncation (nine modes used), but no estimation errors.

(b) Stress estimation: based on the estimated modal parameters from the data. Contains modal truncation and estimation errors.

(c) Statistical $\chi^{2}$ tests on stress: based on the estimated modal parameters from the data and their uncertainties. Contains modal truncation, but compensates for estimation errors.

\subsubsection{Theoretic, deterministic and statistical results at one $s$-value}

In a first example, the computation of the stress at one $s$-value only is demonstrated for the $50 \%$ damage case in element 66 . In Figure 2 the results are shown for $s=1+140 \mathrm{i}$. It can be seen that the stress computation from the
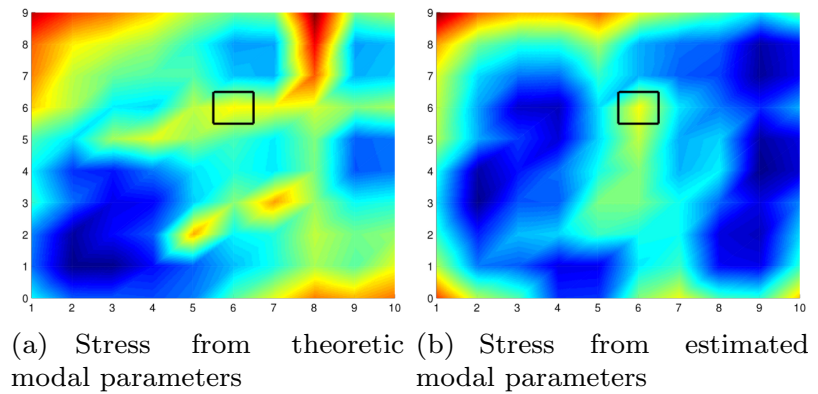
modal parameters modal parameters

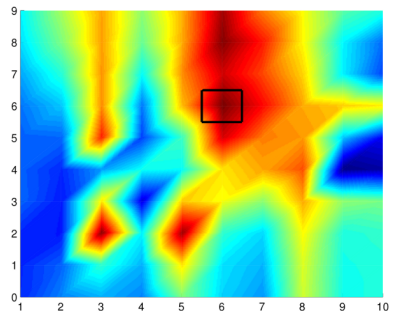

(c) New statistical evaluation of estimated stress

Figure 2: Localization results of plate simulation: stress computation and statistical evaluation at $s=1+140 \mathrm{i}$ (50\% damage in element 66$)$. 


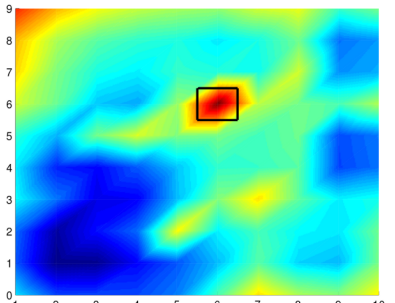
(a) Stress from theoretic
modal parameters

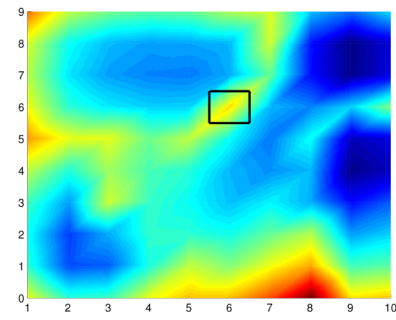

(b) Stress from estimated modal parameters

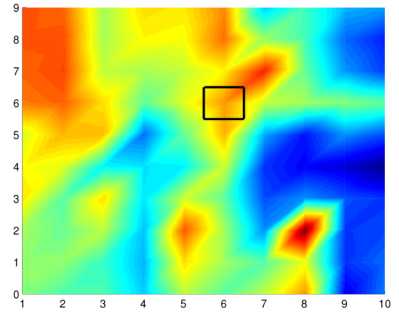

(c) New statistical evaluation of estimated stress

Figure 3: Localization results of plate simulation: stress computation and statistical evaluation at $s=1+800$ i (50\% damage in element 66 ).

exact modal parameters in (a) cannot indicate the damage position, due to modal truncation. The same happens for the computation from the estimated modal parameters in (b). However, when taking into account the measurement uncertainties in the statistical evaluation in (c), the damage location is found amongst (several) $\chi^{2}$ values that are close to zero.

In Figure 3, $s=1+800 \mathrm{i}$ was used for the computation. Note that the damage is now localized perfectly, when the true modal parameters are known in (a). However, the small changes in the estimated modal parameters due to noise in (b) already lead to errors in the stress computation that prevent the localization. Taking into account the estimation uncertainties in (c) improves the situation only slightly. Note that the results shown in (a) are purely theoretic and can never be achieved on real data.

\subsubsection{Joint stress evaluation for damage localization}

From the results in the previous section it can be concluded that localization with the SDDLV is sometimes not successful due to modal truncation, even when the modal parameters are exact, thus motivating the use of more than one $s$ value for the stress evaluation. Furthermore, even small estimation errors of the modal parameters can further degrade the stress results. Then, considering the statistical uncertainties of the modal parameters for the subsequent stress evaluation can improve the situation significantly, since the variance errors on 
the estimation are taken into account and the estimated parameters are weighted in a sensible way.

Hence, we perform a joint stress evaluation at all six $s$-values that are defined above, taking into account the modal parameter uncertainties. In Figure 4, the results are shown for the $30 \%$ damage case. In this case the damage is found in the neighbouring elements of the damaged element 66 with the statistical evaluation in (c), but not precisely at element 66 due to the low level of damage, while the detected damage is far off without the statistical evaluation even with the exact modal parameters in (a) and with the modal parameter estimates in (b). With $50 \%$ damage in element 66 , the localization is successful with the statistical stress evaluation in Figure 5 in (c). The damage can be precisely located, while this is not the case without the statistical evaluation.

Note that in all cases the global damage to the simulated structure is very low with less than $0.8 \%$ frequency decrease after damage. With very small damage, the estimate of the surrogate of the transfer matrix difference $\delta R(s)$ deteriorates significantly due to noise, since a substraction of two noisy matrices from reference and damaged states is made that are close when the change is small. Still, at this level of damage we were able to localize damage based on the SDDLV method, where the statistical evaluation of the results as well as taking several $s$-values for evaluation proved to be crucial for a precise and robust localization.
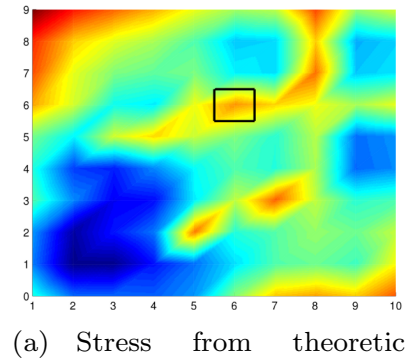
modal parameters

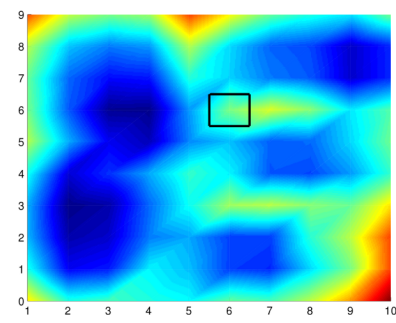

(b) Stress from estimated modal parameters

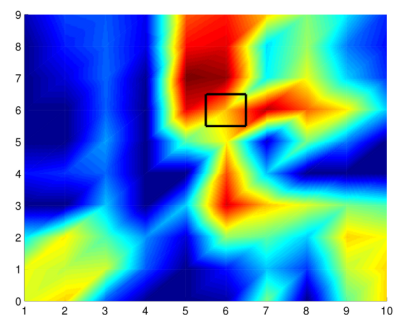

(c) New statistical evaluation of estimated stress

Figure 4: Localization results of plate simulation: joint stress computation and statistical evaluation at six $s$-values (30\% damage in element 66 ). 


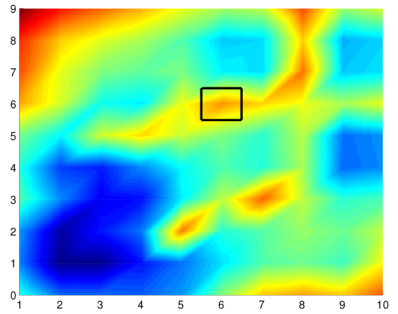

(a) Stress from theoretic modal parameters

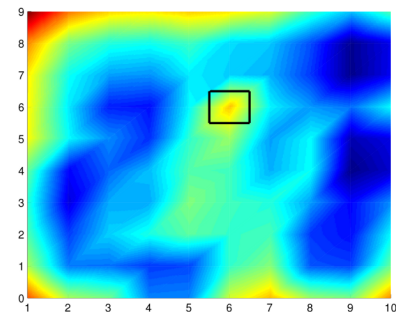

(b) Stress from estimated modal parameters

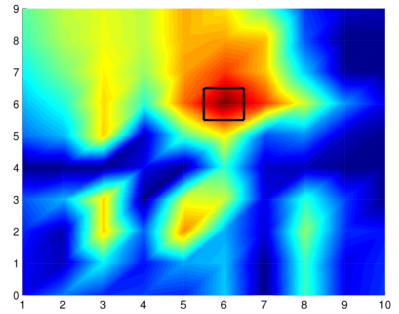

(c) New statistical evaluation of estimated stress

Figure 5: Localization results of plate simulation: joint stress computation and statistical evaluation at six $s$-values (50\% damage in element 66 ).

\subsection{Real beam experiment}

\subsubsection{Experimental setup and measurements}

In a lab experiment, damage tests were conducted on a beam (Figure 6) by Brüel \& Kjær as a benchmark for damage localization. The considered beam is made of PVC. Its dimensions are $50 \mathrm{~cm} \times 8 \mathrm{~cm} \times 1 \mathrm{~cm}$ and it is fixed on one side. For both the undamaged and the damaged states, acceleration data of length $N=295,936$ with a sampling frequency of $8192 \mathrm{~Hz}$ was recorded under white noise excitation by a shaker. The available output data was downsampled and decimated by factor 6 , while the shaker input was not used in the subsequent analysis. The beam has been equipped with 27 sensors on the top and on the bottom (see Figure 6) as follows:

- Sensors $1,4,7, \ldots, 25$ are installed on the top of the beam and are vertical accelerometers.

- Sensors $2,5,8, \ldots, 26$ are installed on the top of the beam and are horizontal accelerometers.

- Sensors $3,6,9, \ldots, 27$ are installed on the bottom of the beam and are horizontal accelerometers. 
Since the shaker acts in the horizontal direction, the vertical accelerometers are dismissed and only the 18 accelerometers in horizontal direction are used for the following damage localization.

A FEM of the beam has been made consisting of 72 plate elements as depicted in Figure 7, from which matrix $\mathcal{L}_{\text {model }}(s)$ is obtained at the chosen Laplace variables. Note that the beam from Figure 6 is rotated for convenience in the model in Figure 7, where the sensors on the top are now on the bottom and vice versa.

The beam was damaged by drilling holes, which are located in the region indicated in Figure 7. In a first scenario, three holes were drilled, and the damaged was increased to 5 holes in a second scenario around the same location. For drilling each new hole, the beam was removed and repositioned, which led to a slight change of the shaker position in each experiment. This introduced a mass change at the shaker position in the vicinity of element P23 between the undamaged and the damaged states. Damage localized in this area should therefore be discarded, which would not appear in real operational cases.

\subsubsection{Modal analysis and uncertainties}

From the measurement data, eight well-estimated modes were chosen in the undamaged and the two damaged states with the procedure described in Section 3. The corresponding frequencies and their coefficients of variation are shown

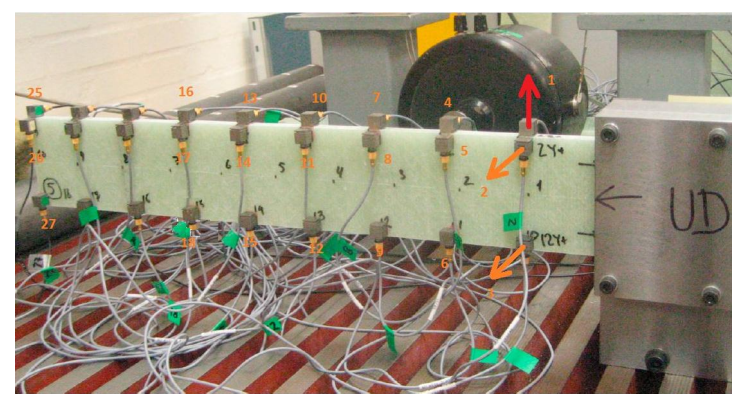

Figure 6: Experimental setup of the beam.

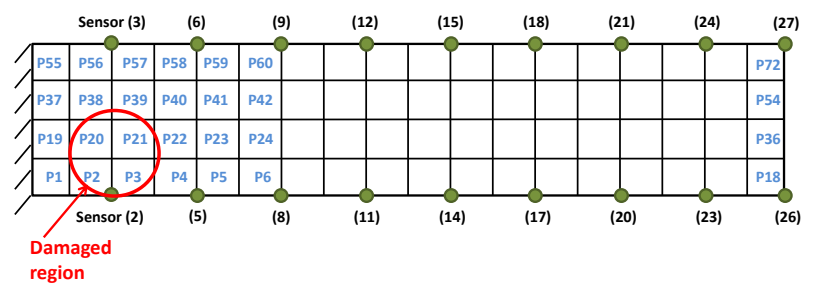

Figure 7: Model of the beam with 18 sensors and the damage location. 
in Table 2 for each chosen mode. Then, the matrices $\widehat{A}_{c}$ and $\widehat{C}_{c}$ and their covariances are obtained in the different states.

\subsubsection{Joint stress computation and statistical evaluation}

The stress resultants and their covariances are computed for two different sets of Laplace variables $s_{i}$ of different size, one with four values $s_{i} \in\{1+3 \mathrm{i}, 1+$ $110 \mathrm{i}, 1+350 \mathrm{i}, 1+520 \mathrm{i}\}$ and another one with ten values $s_{i} \in\{1+3 \mathrm{i} ; 1+50 \mathrm{i} ; 1+$ $110 \mathrm{i} ; 1+350 \mathrm{i} ; 1+520 \mathrm{i} ; 1+700 \mathrm{i} ; 1+855 \mathrm{i} ; 1+900 \mathrm{i} ; 1+940 \mathrm{i} ; 1+1600 \mathrm{i}\}$. Then, the joint stress evaluation for localization is performed with the new statistical method (Section 4.5) and compared to the deterministic aggregation (Section 2.2). The results are shown in Figures 8 and 9 for both sets of Laplace variables, respectively.

In both figures it can be seen that the damage localization procedure with the new statistical evaluation (Figures (b), (d)) indicates strongly damage at the true damage location and sometimes in the directly adjacent elements, while the localization based on the deterministic aggregation of the stress results (Figures (a), (c)) is not successful. The use of more Laplace variables for the statistical

Table 2: Identified frequencies (in $\mathrm{Hz}$ ) of beam with coefficients of variation (in $\%)$.

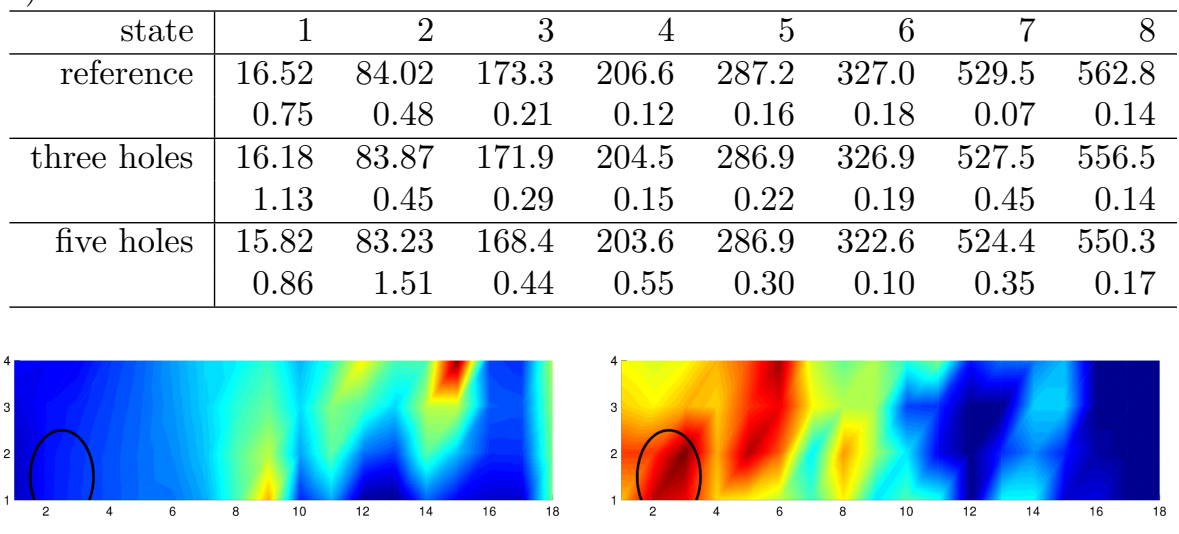

(a) Stress computation, three holes.

(b) New statistical evaluation, three holes.

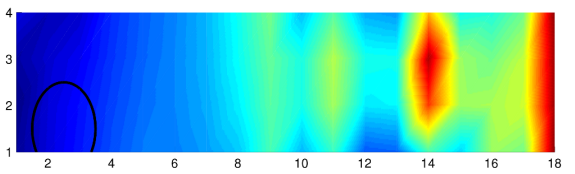

(c) Stress computation, five holes.

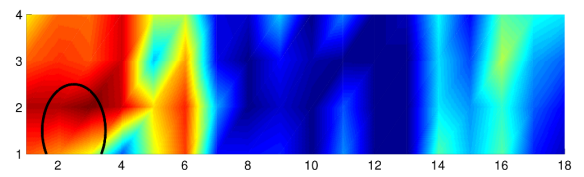

(d) New statistical evaluation, five holes.

Figure 8: Localization results of beam experiment: joint stress computation and statistical evaluation at four $s$-values and different damage levels. 
aggregation of the stress results leads to clearer localization results in Figures 9 (b) and 9(d), compared to Figures 8(b) and 8(d), respectively.

The aggregated results that are shown in both figures often spread within the vertical direction of the beam, since damages at the same horizontal, but at different vertical coordinates are hard to distinguish with the used sensor and shaker layout. Note that the minimal test values are still found in the lower part of the beam, correctly indicating the damage location with the new procedure, while the small extent of the damage, its proximity to the fixed part of the beam and the lack of sensors on the vertical axis impose difficulties for the localization approach. Note also that the change in the shaker position between the reference and damaged states is clearly visible as a damage towards the middle of the beam (corresponding to elements in the vicinity of P23), which should be discarded as explained above.

Summarizing the results, it is found that the deterministic aggregation of the stress vectors from the SDDLV approach could not provide sufficient resolution for damage localization in this experiment, while the statistical evaluation and aggregation of the same stress vectors with the new method described in this paper made a correct damage localization possible. As shown in the plate simulation in Section 5.1, failure of the deterministic aggregation might be due to modal truncation and estimation errors, while the statistical evaluation takes the estimation uncertainty into account. The additional uncertainty information provides an intrinsic weighting for the stress resultants corresponding to the uncertainty of the different modes as well as the different $s$-values, improving the damage localization results.

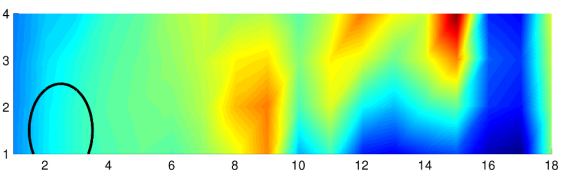

(a) Stress computation, three holes.

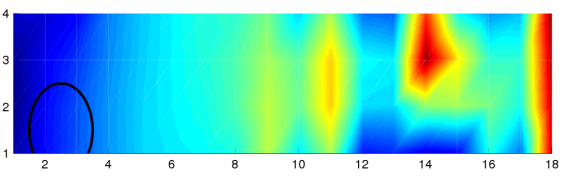

(c) Stress computation, five holes.

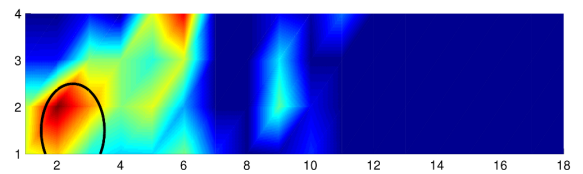

(b) New statistical evaluation, three holes.

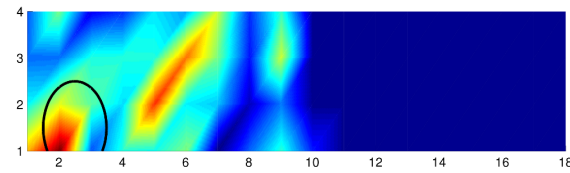

(d) New statistical evaluation, five holes.

Figure 9: Localization results of beam experiment: joint stress computation and statistical evaluation at ten $s$-values and different damage levels. 


\section{CONCLUSION}

In this paper, we have formalized a damage localization approach combining structural model information and data-driven uncertainties computation. The main focus has been the robustness of the method with respect to the fusion of damage localization results computed at different Laplace variables in the complex plane. The selection of these Laplace variables itself is not addressed in this paper. It has been shown how the additional uncertainty information obtained from measurements is useful for damage localization. The resulting statistical evaluation removes a part of the estimation errors and uncertainty related to the choice of the Laplace variables, which leads to a more robust damage localization than with the deterministic evaluation as previously done. The approach has been applied on relevant examples, both on numerical simulations and on experimental data from a case study in the lab. Future works should address further important parts of the underlying damage localization framework, such as the number and choice of the Laplace variables, the effect of modal truncation on the estimates and multiple damages, the optimal number, position and kind of sensors, etc. Furthermore, the method should be applied on structures in the field, where the FEM size and the identification parameterization are largely different.

\section{ACKNOWLEDGMENTS}

This work was partially supported by the European project FP7-PEOPLE-2009IAPP 251515 ISMS, which is gratefully acknowledged. Furthermore, we thank Brüel \& Kjær for providing the data from the beam experiment.

\section{References}

[1] W. Heylen, S. Lammens, P. Sas, Modal Analysis Theory and Testing, Katholieke Universiteit Leuven, Belgium, 1998.

[2] B. Peeters, G. De Roeck, Stochastic system identification for operational modal analysis: a review, Journal of Dynamic Systems, Measurement, and Control 123 (4) (2001) 659-667.

[3] E. Carden, P. Fanning, Vibration based condition monitoring: a review, Structural Health Monitoring 3 (4) (2004) 355-377.

[4] W. Fan, P. Qiao, Vibration-based damage identification methods: a review and comparative study, Structural Health Monitoring 10 (1) (2011) 83-111.

[5] J. M. W. Brownjohn, P.-Q. Xia, H. Hao, Y. Xia, Civil structure condition assessment by FE model updating: methodology and case studies, Finite Elements in Analysis and Design 37 (10) (2001) 761-775. 
[6] M. Basseville, L. Mevel, M. Goursat, Statistical model-based damage detection and localization: subspace-based residuals and damage-to-noise sensitivity ratios, Journal of Sound and Vibration 275 (3) (2004) 769-794.

[7] E. Balmès, M. Basseville, L. Mevel, H. Nasser, W. Zhou, Statistical modelbased damage localization: a combined subspace-based and substructuring approach, Structural Control and Health Monitoring 15 (6) (2008) 857-875.

[8] M. Döhler, L. Mevel, F. Hille, Efficient computation of minmax tests for fault isolation and their application to structural damage localization, in: Proc. 19th IFAC World Congress, Cape Town, South Africa, 2014.

[9] D. Bernal, Load vectors for damage localization, Journal of Engineering Mechanics 128 (1) (2002) 7-14.

[10] Z. Duan, G. Yan, J. Ou, B. F. Spencer, Damage detection in ambient vibration using proportional flexibility matrix with incomplete measured dofs, Structural Control and Health Monitoring 14 (2) (2007) 186-196.

[11] E. Reynders, G. De Roeck, A local flexibility method for vibration-based damage localization and quantification, Journal of Sound and Vibration 329 (12) (2010) 2367-2383.

[12] D. Bernal, Load vectors for damage location in systems identified from operational loads, Journal of Engineering Mechanics 136 (1) (2010) 31-39.

[13] R. Pintelon, P. Guillaume, J. Schoukens, Uncertainty calculation in (operational) modal analysis, Mechanical Systems and Signal Processing 21 (6) (2007) 2359-2373.

[14] E. Reynders, R. Pintelon, G. De Roeck, Uncertainty bounds on modal parameters obtained from stochastic subspace identification, Mechanical Systems and Signal Processing 22 (4) (2008) 948-969.

[15] E. Carden, A. Mita, Challenges in developing confidence intervals on modal parameters estimated for large civil infrastructure with stochastic subspace identification, Structural Control and Health Monitoring 18 (1) (2011) 5378.

[16] S. Sankararaman, S. Mahadevan, Uncertainty quantification in structural damage diagnosis, Structural Control and Health Monitoring 18 (8) (2011) $807-824$.

[17] S. Sankararaman, S. Mahadevan, Bayesian methodology for diagnosis uncertainty quantification and health monitoring, Structural Control and Health Monitoring 20 (1) (2013) 88-106.

[18] M. Döhler, L. Marin, D. Bernal, L. Mevel, Statistical decision making for damage localization with stochastic load vectors, Mechanical Systems and Signal Processing 39 (1-2) (2013) 426-440. 
[19] B. Peeters, G. De Roeck, Reference-based stochastic subspace identification for output-only modal analysis, Mechanical Systems and Signal Processing 13 (6) (1999) 855-878.

[20] A. Benveniste, L. Mevel, Nonstationary consistency of subspace methods, IEEE Transactions on Automatic Control AC-52 (6) (2007) 974-984.

[21] M. Döhler, L. Mevel, Fast multi-order computation of system matrices in subspace-based system identification, Control Engineering Practice 20 (9) (2012) 882-894.

[22] M. Döhler, L. Mevel, Efficient multi-order uncertainty computation for stochastic subspace identification, Mechanical Systems and Signal Processing 38 (2) (2013) 346-366.

[23] D. Bernal, Flexibility-based damage localization from stochastic realization results, Journal of Engineering Mechanics 132 (6) (2006) 651-658.

[24] D. Bernal, Damage localization from the null space of changes in the transfer matrix, AIAA Journal 45 (2) (2007) 374-381.

[25] E. Reynders, J. Houbrechts, G. De Roeck, Fully automated (operational) modal analysis, Mechanical Systems and Signal Processing 29 (2012) 228250.

[26] M. Döhler, X.-B. Lam, L. Mevel, Uncertainty quantification for modal parameters from stochastic subspace identification on multi-setup measurements, Mechanical Systems and Signal Processing 36 (2013) 562-581. 